\title{
Effet d'aliments à base de pomme cajou sur les performances pondérale et la carcasse des canetons en croissance au Bénin
}

\author{
André Boya $\mathrm{ABOH}^{1 *}$, Jacques T. DOUGNON ${ }^{2}$, Ghislaine S. Théodora ATCHADE ${ }^{1}$ \\ et André M. TANDJIEKPON ${ }^{3}$ \\ ${ }^{1}$ Laboratoire des Recherches Zootechnique, Vétérinaire et Halieutique (LRZVH), Centre de Recherches \\ Agricoles d'Agonkanmey (CRA-AgonKanmey), Institut National des Recherches Agricoles du Bénin \\ (INRAB), 01 BP 2359 Recette Principale, Cotonou République du Bénin ; \\ Institut National des Recherches Agricoles du Bénin, 01 BP 884 Cotonou, Bénin. \\ ${ }^{2}$ Laboratoire de Recherches en Biologie Appliquée (LARBA), Ecole Polytechnique d'Abomey-Calavi à \\ l'Université d'Abomey-Calavi (UAC), 01 BP 2009 Cotonou, Bénin. \\ ${ }^{3}$ Programme de Recherche Forestière, Institut National des Recherches Agricoles du Bénin, \\ 01 BP 884, Cotonou, Bénin. \\ *Auteur correspondant,E-mail :a2abohboya@yahoo.fr / aboh.solex@gmail.com; Tel :00 22997931422.
}

\section{RESUM}

La pomme cajou (PC) constitue un sous-produit agricole disponible au Bénin en quantité et sans une importance économique. Pour la conservation de cette matière première, le séchage solaire est réalisé avant son incorporation dans l'aliment concentré des canetons de barbarie. Les rations alimentaires testées R0, R10, R20 et R30 comportaient respectivement $0,10,20$ et $30 \%$ de PC séchée. L'expérimentation a concerné 64 canetons âgés de 10 à 21 jours. Le séchage solaire de la pomme cajou a permis sa conversion en un produit stabilisé ayant une valeur alimentaire intéressante. La consommation journalière d'aliment à base de la PC séchée a varié de 67 à $150 \mathrm{~g}$ MS/canard. Les meilleurs gains de poids et indices de consommation sont obtenus chez les canards alimentés avec les rations R10 et R20. Le rendement en carcasse a varié de 66 à 69,5\% chez les oiseaux. Le recyclage de la PC dans l'alimentation des canetons,constitue alors une technologie d'appoint pour les agro-éleveurs en vue de la réduction du coût élevé des aliments et de la valorisation de ce sous- produit. (c) 2011 International Formulae Group. All rights reserved.

Mots clés : Pomme cajou, canard, gain de poids, indice de consommation, carcasse.

\section{INTRODUCTION}

Dans le cadre de la politique de diversification des cultures, la production de l'anacarde s'est intensifiée ces dernières années au Bénin. Sa production permet de résoudre les problèmes complémentaires de développement tels que l'économie, le social et l'environnement.
L'ouverture du marché d'exportation de la noix de cajou vers l'extérieur a accrû la production de l'anacarde. Elle est en moyenne de 50.000 tonnes par an dont la presque totalité est exportée vers l'extérieur. Quant à la production de la pomme cajou (faux fruit), elle est estimée à 200.000 tonnes par an (Tandjiékpon et al., 2003). Malheureusement, à la récolte de la noix, la pomme cajou est 
délaissée dans les champs sans une valorisation appropriée. Jusqu'à présent la consommation de la PC brute ou sous la forme transformée ne dépasse pas $10 \%$ de la production totale. $\mathrm{Ce}$ qui indique pratiquement une perte post-récolte annuelle d'environ $90 \%$ de la production.

Outre la teneur en eau (86\%), la PC présente une teneur élevée en tanin qui limite la conservation pour des fins utiles (Morton, 1987 ; Vijayakumar, 1991). Toutefois, elle est transformée dans certains pays en plusieurs produits tels que le jus, la confiture, le vin, le vinaigre et l'alcool (Vijayakumar, 1991). La valeur alimentaire de la PC en pourcentage de matière sèche $(12,32 \% \mathrm{MS})$ est de l'ordre de 6,45 à $12,5 \%$ de protéines brutes, 3,5 à $10,85 \%$ de fibre totale, 30,6 à $54,7 \%$ de sucre total (glucose), 30,8 mg/100 g de vitamine $\mathrm{C}$ et de $12,48 \mathrm{MJ} / \mathrm{kg}$ d'énergie et des minéraux (Morton, 1987 ; Lakshmipathi et al., 1990).

La sous-alimentation constitue l'une des causes majeures de la faible productivité de canetons de barbarie, malgré la disponibilité de cette ressource alimentaire importante. Les études sur l'utilisation de la PC comme ressource alimentaire pour la volaille sont limitées et n'abordent pas son effet sur la carcasse (Lakshmipathi et al., 1990 ; Song et Seng, 2008).

La présente étude a pour objectif d'investiguer sur l'effet des concentrés alimentaires à base de PC sur la croissance pondérale, la santé et les caractéristiques de la carcasse du canard de barbarie.

\section{MATERIEL ET METHODES}

\section{Collecte des pommes cajou et rations expérimentales}

Les pommes cajou mûres ont été collectées après la récolte des noix cajou. Ces PC ont été séchées au soleil à une température moyenne de $30{ }^{\circ} \mathrm{C}$. Elles ont été ensuite incorporées à des proportions de $0,10,20$ et $30 \%$ aux autres matières premières et broyées pour obtenir des rations alimentaires farineuses contenant quelques grains R0, R10, $\mathrm{R} 20$ et $\mathrm{R} 30$ respectivement (Tableau 1).

Analyse chimique des aliments

Les échantillons de la PC et des concentrés alimentaires ont été prélevés pour la détermination de la matière sèche à l'étuve, à la température de $70{ }^{\circ} \mathrm{C}$ jusqu'à poids constant. L'analyse chimique des aliments a concerné l'azote $(\mathrm{N})$ déterminé par la méthode Kjeldhal, le phosphore (P), le Calcium (Ca) et le Magnésium $(\mathrm{Mg})$ suivant la procédure AOAC (1990). L'azote déterminé a été multiplié par 6,25 pour calculer la Matière Azotée Totale (MAT).

\section{Conduite de l'expérimentation}

Un effectif de 64 canetons de barbarie âgés de 10 à 21 jours a été utilisé pour l'expérimentation qui a duré 84 jours après 10 jours d'adaptation alimentaire. Le poids vif corporel individuel moyen était de $355 \mathrm{~g}$ au début de l'expérimentation. L'alimentation de groupe a été utilisée pour l'expérimentation. Ainsi, les canetons ont été répartis en 4 lots homogènes de 16 sujets. Chaque lot composé de 4 groupes de 4 canetons ( 3 femelles et 1 mâle) a été affecté au hasard à l'une des quatre rations. Chaque groupe de canetons est placé dans des enclos de $2 \mathrm{~m}$ de long, 1,2 m de large et $0,7 \mathrm{~m}$ de haut dont la litière est constituée de copeaux de bois. La quantité d'aliment servie par sujet a varié de $80 \mathrm{~g}$ à $210 \mathrm{~g}$ MS et l'eau est distribuée à volonté. Les canetons ont été déparasités avec le VPV® (Lévamisole) et la prévention de la coccidiose était faite avec l'Anticox® (Sulfadimidine sodique et Diavéridine).

Au cours de l'expérimentation, le point des quantités d'aliments servies et refusées (en MS) a été enregistré tous les 14 jours. Le poids vif corporel individuel a été pris tous les 14 jours. Sur le plan sanitaire, les signes cliniques des maladies apparues ainsi que le nombre de canetons morts au cours de l'expérimentation ont été enregistrés. 
Tableau 1 : Composition centésimale des rations alimentaires testées.

\begin{tabular}{|c|c|c|c|c|}
\hline \multirow[t]{2}{*}{ Ingrédients alimentaires } & \multicolumn{4}{|c|}{ Rations alimentaires } \\
\hline & R0 & R10 & $\mathrm{R} 20$ & R30 \\
\hline Pomme cajou & 0 & 10 & 20 & 30 \\
\hline Maïs & 31,5 & 26 & 17,5 & 10 \\
\hline Son de blé & 47 & 40 & 36 & 31 \\
\hline Tourteaux de coton & 10 & 10 & 10 & 10 \\
\hline Tourteaux de soja & 8 & 10,5 & 13 & 15,5 \\
\hline Coquille d'huitre & 2,5 & 2,5 & 2,5 & 2,5 \\
\hline Prémix $(\mathrm{CMV})^{1}$ & 0,5 & 0,5 & 0,5 & 0,5 \\
\hline Sel & 0,5 & 0,5 & 0,5 & 0,5 \\
\hline \multicolumn{5}{|c|}{ Composition chimique (calculée en \% MS) } \\
\hline Energie métabolisable ( $\mathrm{kcal} / \mathrm{kg})$ & 2845 & 2980 & 3859 & 4957 \\
\hline Protéines Brutes & 13.87 & 14.1 & 14.7 & 15.1 \\
\hline Fibre totale & 6.27 & 6.67 & 7.33 & 7.91 \\
\hline $\mathrm{Ca}$ & 1.07 & 1.07 & 1.07 & 1.07 \\
\hline $\mathrm{P}$ & 0.76 & 0.56 & 0.62 & 0.68 \\
\hline
\end{tabular}

L'évaluation de la carcasse, a porté sur deux canards par ration alimentaire qui ont été abattus à la fin de l'essai. L'observation a porté sur le poids, l'appréciation visuelle, le toucher de la carcasse et des organes tels que le foie, le poumon, le cœur, le gésier, l'intestin, la trachée, l'œsophage pour apprécier la couleur, la consistance et le volume.

\section{Analyse statistique}

Les données sur la consommation, le poids vif, le gain de poids et l'indice de consommation sont soumises à une analyse de variance avec le logiciel Statistica 6.0 (1998). En cas de différence significative, le test de Student Newman-Keuls a été utilisé pour séparer les groupes homogènes en fonction des rations alimentaires.

\section{RESULTATS}

\section{Composition chimique des aliments}

Le taux d'humidité de la PC fraîche était de $86 \%$ et celui de la matière sèche de la PC séchée à l'air libre était de 77\%. La teneur en Matière Azotée Totale (MAT) de la PC était de 9,6\%. Celle des rations alimentaires a varié de 19 à 20,5\% (Tableau 2).

\section{Consommation alimentaire}

La consommation alimentaire a varié de $\quad 67$ à $150 \quad \mathrm{~g} \quad \mathrm{MS} / \mathrm{j}$ (Figure 1). La consommation moyenne est plus élevée $(\mathrm{P}<$ 0,01 ) pour la ration alimentaire R20, suivie de R30 (Figure 1).

\section{Etat sanitaire des canards}

En général, les canards ont présenté un état sanitaire apparemment sain. Toutefois, la plupart des oiseaux soumis au régime alimentaire contenant de la PC ont fait une quinte de toux au moment précis de la consommation de la ration alimentaire. Cette quinte de toux disparaît juste à la fin de la consommation alimentaire. Les taux de mortalité enregistrés ont été de $6,25 \%$ chez les canetons nourris avec les rations alimentaires contenant ou non la PC.

\section{Performance pondérale des canards}

Les résultats de la Figure 2 montrent que la croissance pondérale a été plus élevée 
( $\mathrm{p}<0,05)$ chez les canards soumis au régime alimentaire R20. Concernant le gain de poids moyen quotidien, il a varié de 12,2 à 16,8 g (Tableau 3). Ce gain de poids a été plus élevé $(\mathrm{p}<0,01)$ chez les canards nourris à la ration alimentaire R20.

Quant à l'indice de consommation, il a varié de 6,7 à 8,2 (Tableau 3). Les plus faibles indices de consommation $(\mathrm{p}<0,05)$ ont été obtenus chez les canards nourris aux rations alimentaires R20 et R10.

\section{Appréciation des carcasses et viscères}

A l'observation et au touché, le volume et la consistance du foie, du poumon, du cœur et du gésier étaient similaires à ceux des canards alimentés avec la ration témoin sans PC. Par ailleurs, la couleur de ces organes, ainsi que celle du sang et du muscle présentaient les mêmes caractéristiques que celles des canards alimentés à la ration témoin. Le poids de la carcasse a varié de 853 à 1078 g (Tableau 4); ce qui correspondait à un rendement carcasse variant de 66 à $70 \%$. Les viscères consommables et non consommables représentaient respectivement 24 à $27 \%$ et 5 à $6 \%$ du poids vif du canard.

Tableau 2 : Teneur en matière azotée totale (MAT \% MS), phosphore (P \% MS), calcium (Ca \% $\mathrm{MS}$ ) et magnésium (Mg \% MS) de la pomme cajou et des rations alimentaires.

\begin{tabular}{lcccc}
\hline Ingrédients/rations alimentaires & MAT & P & Ca & Mg \\
\hline Pomme cajou & 9,56 & 0,23 & 0,64 & 0,19 \\
R0 & 19,00 & 0,71 & 1,04 & 0,53 \\
R10 & 20,56 & 0,79 & 1,20 & 0,75 \\
R20 & 19,75 & 0,80 & 1,04 & 0,82 \\
R30 & 18,94 & 0,85 & 1,28 & 0,67
\end{tabular}

R0 : ration alimentaire contenant $0 \%$ de la pomme cajou séchée ; R10 : ration alimentaire contenant $10 \%$ de la pomme cajou séchée ; R20 : ration alimentaire contenant $20 \%$ de la pomme cajou séchée ; R30 : ration alimentaire contenant $30 \%$ de la pomme cajou séchée.

Tableau 3 : Gain de poids moyen quotidien (GMQ) et indice de consommation (IC).

\begin{tabular}{lcc}
\hline Ration alimentaire & GMQ $(\mathbf{g} / \mathbf{j})$ & IC \\
\hline R0 & $12,2 \mathrm{c}$ & $7,8 \mathrm{a}$ \\
R10 & $14,8 \mathrm{~b}$ & $6,8 \mathrm{~b}$ \\
R20 & $16,8 \mathrm{a}$ & $6,6 \mathrm{~b}$ \\
R30 & $13,7 \mathrm{~b}$ & $8,2 \mathrm{a}$ \\
\hline P & 0,008 & 0,011
\end{tabular}

Les moyennes de la même colonne suivies de lettres différentes sont significativement différentes selon le test de Student-Newman-Keuls au seuil de probabilité de $5 \%$.

R0 : ration alimentaire contenant $0 \%$ de la pomme cajou séchée ; R10 : ration alimentaire contenant $10 \%$ de la pomme cajou séchée ; R20 : ration alimentaire contenant $20 \%$ de la pomme cajou séchée ; R30 : ration alimentaire contenant $30 \%$ de la pomme cajou séchée. 
A. B. ABOH et al. / Int. J. Biol. Chem. Sci. 5(6): 2407-2414, 2011

Tableau 4 : Variation du poids des carcasses (en g) et des viscères (en g) des canards.

\begin{tabular}{lccccccccc}
\hline Ration alimentaire & \multicolumn{2}{c}{ Carcasse } & \multicolumn{3}{c}{ Viscères consommables } & \multicolumn{3}{c}{ Viscères non consommables* } \\
\hline & Poids & $\%$ & Foie & Poumon & Cour & Gésier & $\%$ & Poids & $\%$ \\
R0 & $853 \pm 308$ & 66,3 & $23 \pm 0,7$ & $8 \pm 2,1$ & $9 \pm 4,2$ & $27 \pm 9,8$ & 24,7 & $67 \pm 21$ & 6,0 \\
R 10 & $962 \pm 89,1$ & 69,5 & $25 \pm 2,1$ & $10 \pm 2,1$ & $14 \pm 0,7$ & $37 \pm 8,5$ & 24,1 & $88 \pm 4,2$ & 6,4 \\
R 20 & $1078 \pm 23,3$ & 68,5 & $33 \pm 6,4$ & $10 \pm 0,7$ & $13 \pm 0,7$ & $39 \pm 2,8$ & 26,4 & $77 \pm 1,4$ & 4,9 \\
R 30 & $979 \pm 5,7$ & 67,3 & $29 \pm 0,0$ & $12 \pm 0,7$ & $14 \pm 0,7$ & $35 \pm 5,0$ & 27,3 & $78 \pm 2,8$ & 5,4 \\
\hline
\end{tabular}

* = Intestins, trachée, œsophage, rate ; R0 : ration alimentaire contenant $0 \%$ de la pomme cajou séchée ; R10 : ration alimentaire contenant 10\% de la pomme cajou séchée ;

R20 : ration alimentaire contenant $20 \%$ de la pomme cajou séchée ; R30 : ration alimentaire contenant 30\% de la pomme cajou séchée.

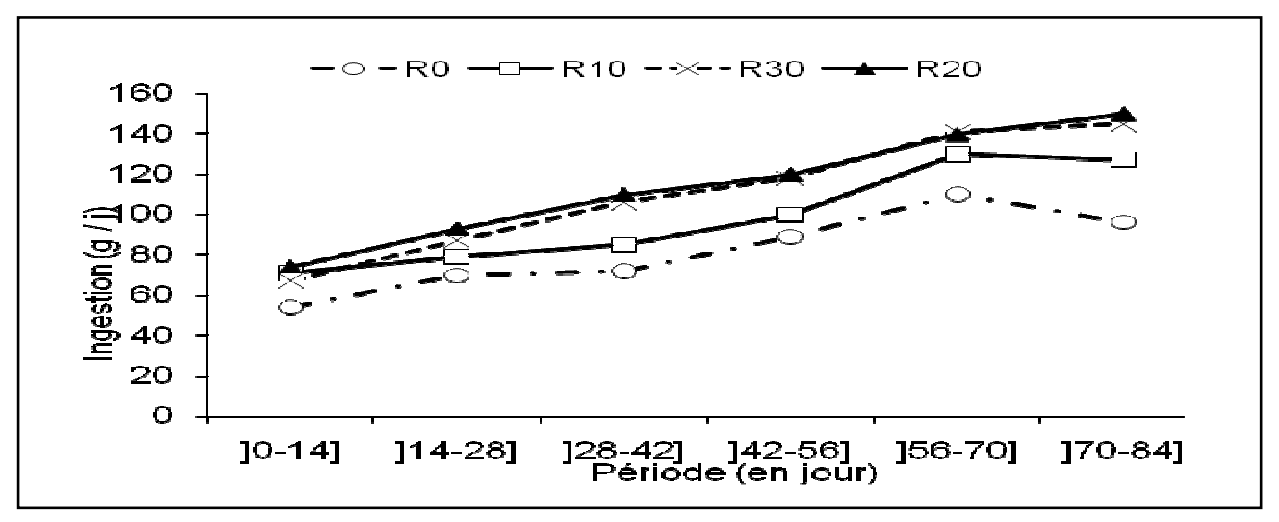

Figure 1 : Evolution de la consommation des rations alimentaires (g MS/j) chez les canards de barbarie.

R0 : ration alimentaire contenant $0 \%$ de la pomme cajou séchée ; R10 : ration alimentaire contenant $10 \%$ de la pomme cajou séchée :

R20 : ration alimentaire contenant $20 \%$ de la pomme cajou séchée ; R30 : ration alimentaire contenant $30 \%$ de la pomme cajou séchée. 


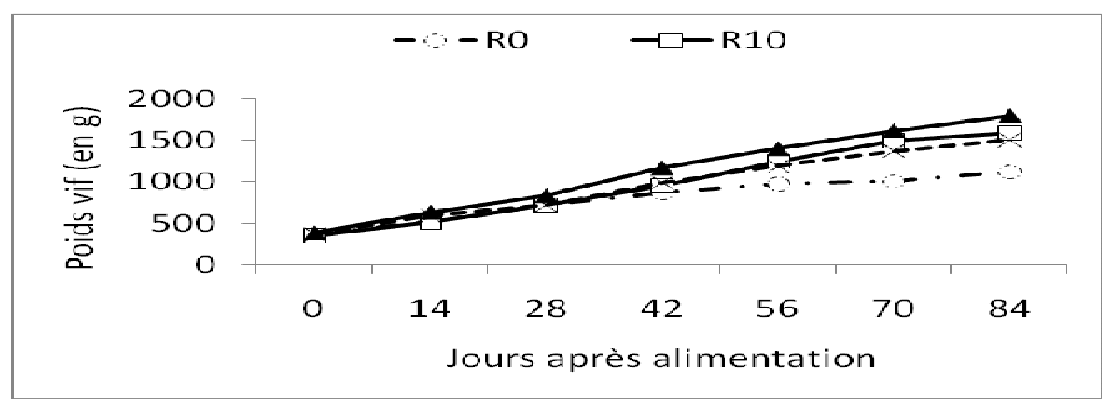

Figure 2 : Evolution du poids vif $(\mathrm{g} / \mathrm{j})$ des canards de barbarie.

R0 : ration alimentaire contenant $0 \%$ de la pomme cajou séchée; R10 : ration alimentaire contenant $10 \%$ de la pomme cajou séchée ; R20 : ration alimentaire contenant $20 \%$ de la pomme cajou séchée ; R30 : ration alimentaire contenant $30 \%$ de la pomme cajou séchée.

\section{DISCUSSION}

Valeur alimentaire de la pomme cajou et d'aliment concentré

Le séchage solaire de la PC permet la conversion de la denrée périssable en un produit stabilisé par déshydratation. Ce qui éviterait les attaques microbiennes et la dégradation de la qualité du produit liée aux réactions biochimiques indésirables. Enfin, la conservation par séchage solaire permet de réduire les pertes post-récolte. Ainsi, la valeur alimentaire de la PC séchée est intéressante et comparable à d'autres ingrédients alimentaires. En effet, les teneurs en phosphore et en calcium de la PC séchée sont supérieures à celles du maïs blanc, mais comparables à celles de la drêche de brasserie (Sauvant, 2002; Huart et al., 2003 ). Par ailleurs, la teneur en protéine de la PC est relativement supérieure à $8,5 \%$, valeur rapportée pour le maïs blanc (Sauvant, 2002 ; Huart et al., 2003 ; FAO, 2010). La teneur en protéine brute de la PC rapportée est de 10,5\% (Ogunjobi et Ogunwole, 2010). Cette valeur est relativement élevée par rapport à celle enregistrée dans le cadre de la présente étude. Cet écart serait lié à la différence de la teneur en composition chimique des variétés d'anacardier à la $\mathrm{PC}$ jaune, rouge et orange rapportée par Nguyen Xich et al. (1996). Concernant les teneurs en MAT des rations alimentaires à base de la PC testées, elles sont supérieures à celles suggérées pour les canards de barbarie (Leclercq et de Carville, 1988), soit une teneur en protéines brutes située entre 14 et $15 \%$ pour atteindre d'excellentes performances de croissance chez les canards de barbarie.

\section{Consommation alimentaire et croissance pondérale des canetons}

La mouture de la PC avec les autres matières premières est réalisée avec de mineures difficultés. Le concentré obtenu est un aliment de type farineux comportant quelques grains.

L'expérimentation révèle que les canetons peuvent consommer sans risque d'intoxication majeure, en périodes de croissance et de finition, un aliment contenant la PC séchée. Ce résultat confirme l'utilisation de la PC ou de son sous-produit de transformation comme ingrédient de ration alimentaire chez les monogastriques (Nguyen Xich et al., 1996 ; Fanimo et al., 2003 ; Song et Seng, 2008). Toutefois, l'apparition de quinte de toux chez les canards serait liée à la teneur élevée en tanin ou à l'astringence de la pulpe de la PC (Morton, 1987 ; Vijayakumar, 1991).

Les rations alimentaires à base de la PC ont amélioré les performances pondérales, l'indice de consommation et les caractéristiques de carcasse chez les canetons par rapport à la ration témoin. Cette amélioration de performance zootechnique 
serait liée à l'apport en quantité suffisante par la PC d'oligo-éléments, des acides aminés essentiels, de l'énergie via sa teneur élevée en glucose et des éléments facilitant sa digestion comme la cellulose. La croissance pondérale régulière révèle que les canetons semblent tolérer $20 \%$ de taux d'incorporation de la PC dans l'aliment. Ainsi, concernant les capacités de croissance des oiseaux, il existe donc un taux d'incorporation optimal de la PC qui est $20 \%$ dans le cas de notre expérimentation. Ce taux d'incorporation de la PC dans l'aliment de caneton se rapproche de celui rapporté (15\%) pour le canard Khaki Campbell (Song et Seng, 2008). Par ailleurs, les taux d'incorporation de 20 et $30 \%$ de sous-produits de transformation de la PC sont rapportés pour une meilleure croissance chez les lapins (Fanimo et al., 2003). Le gain de poids obtenu au cours de cette expérimentation, est relativement supérieur à celui enregistré par ce dernier auteur $(15 \mathrm{~g} / \mathrm{j})$. L'indice de consommation enregistré est comparable à celui obtenu dans le Centre Agronomique et Vétérinaire Tropical de Kinshasa et qui varie de 4 à 8,3 (Tona, 2007), mais traduit une faible efficacité des rations testées. En effet, l'indice de consommation obtenu est plus élevé que celui signalé au Cameroun qui varie de 4,3 à 5,7 (Ndjangou et al., 2008). La différence résiderait dans la formule alimentaire qui est peu équilibrée en nutriments.

L'incorporation de la PC dans l'aliment semble induire une augmentation de poids du foie, du poumon, du cœur et du gésier ; mais cette augmentation ne suit pas une tendance logique. L'expérimentation réalisée a montré que le poids du foie et des reins des lapins soumis au régime alimentaire à base de la PC sont significativement supérieurs aux poids de ceux qui ont été soumis à la ration alimentaire sans PC (Fanimo et al., 2003). Les travaux ont démontré que l'accroissement du poids des organes peut être ralentit lorsque les protéines et des acides aminés sont insuffisamment disponibles (Green et al., 1986). C'est probablement ce qui justifie la faible performance enregistrée chez les canards nourris à la ration alimentaire témoin. Toutefois, les rendements des carcasses obtenus chez les canards expérimentaux sont comparables à ceux rapportés (68 à 70,5\%) par Retailleau (1986).

\section{Conclusion}

L'expérimentation montre que le séchage de la PC maintient autant que possible ses propriétés sensorielles sans les altérer. Ainsi, la PC séchée constitue une bonne ressource alimentaire pour les canetons de barbarie. Cet ingrédient alimentaire peut être incorporé à un taux de $20 \%$ dans la ration alimentaire avec un effet positif sur la croissance pondérale et un bon rendement en carcasse des canards. Ainsi, le recyclage de la PC dans l'alimentation des canetons, constitue alors une technologie d'appoint pour les éleveurs en vue de la réduction du coût élevé des aliments; et pour les producteurs d'anacarde en vue de donner une valeur ajoutée à ce produit agricole habituellement jeté. Toutefois, il est important d'approfondir la méthode de traitement de la PC avant son incorporation dans la ration alimentaire afin d'éliminer la quinte de toux observée chez les canards.

\section{REMERCIEMENTS}

Les auteurs remercient sincèrement l'Institut National des Recherches Agricoles du Bénin (INRAB) pour avoir financé ce protocole de recherche à travers le projet danois "Appui à la Recherche Agricole".

\section{REFERENCES}

AOAC (Association of official agricultural chemist) 1990. Official Methods of Analysis (15 ${ }^{\text {th }}$ Edition). Washington DC, USA.

Fanimo AO, Oduguwa OO, Alade AA, Ogunnaike TO , Adesehinwa AK. 2003. Growth performance, nutrient digestibility and carcass characteristic of growing rabbits fed cashew apple waste. Livest. Resear. Rural Dev., 15: 1-7. 
FAO (Food and Agriculture Organization of the United Nations). 2010. Composition of Selected Foods from West Africa, Stadlmayr B, Charrondiere UR, Addy P, Samb B, Enujiugha VN, Bayili RG, Fagbohoun EG, Smith IF, Thiam I, Burlingame B (Eds). FAO; 43.

Green GM, Levan VH, Liddle RA. 1986.Interaction of dietary protein and trypsin inhibitor on plasma cholecystokinin and pancreatic growth in rats. In Nutritional and Toxicological Significance of Enzyme Inhibitors in Foods, Friedmann M (ed). Plenum Press: New York.

Huart A, Leroy P, Thewis A, Bindelle J, Muland M, Kibango D. 2003. La production porcine en République Démocratique du Congo (I). In Troupeaux et cultures des tropiques, Kinshasa. Dossier spécial porc, CAVTK, p. 20.

Lakshmipathi V, Thirumalai S, Vishwanathan MR, Venkatakrishnan R. 1990. Cashew apple-meal as feed for chicks. Indian Jour. Poultry Sc., 25(4): 296-297.

Leclercq B, de Carville H. 1988. Aliments de finition du canard de Barbarie : effets de leurs teneurs en protéines brutes sur les performances et la qualité de carcasse. INRA Prod. Anim., 1(2): 129-131.

Morton J. 1987. Cashew Apple. In Fruits of Warm Climates. FL: Miami.

Ndjangou MH, Teguia A, Dongmo MC, Fuelefac DH, Tchoumboue J. 2008. Effet du taux de protéines brutes de la ration sur les performances de croissance du canard de barbarie (Caïrina moshata). Livest. Resear. Rural Dev., 20(10).

Nguyen Xich L, Lam Qouc A, Nguyen Thi H. 1996. Study of processing and using cashew apple residues as animal's feeds. In Improved the Value of Cashew Apple. Science and Technique Center: Hochiminh, Vietnam; 42-54.
Ogunjobi MAK, Ogunwole SO. 2010. Physicochemical and sensory properties of cassava flour biscuit supplemented with cashew apple powder. J. Food Tech., 8(1): 24-29.

Retailleau B. 1986. La sélection du canard de Barbarie : performances récentes et perspectives. In Comptes rendus de la $3^{\text {ème }}$ Conférence Avicole, Cahier no 3, WPSA-SIMAVIP ; 17-23.

Sauvant D, Perez J-M, Tran G. 2002. Tables de Composition et de Valeurs Nutritives des Matières Premières Destinées aux Animaux d'Elevage: Porcs, Volailles, Bovins, Ovins, Caprins, Lapins, Chevaux, Poissons. Ed INRA ; 301.

Song M, Seng M. 2008. Nutritive value of cashew Apple for growing duck, Poster.

Tropentag, October 7-9, Hohenheim, Germany.

Statistica 6.0. 1998. Logiciel Statistica. Maison Alfort, Paris Statsoft : France.

Tandjiekpon A, Teblekou K, Dah-Dovonon J, N'djolosse K, Adjahuinou L. 2003. La culture de l'anacardier au Bénin. Référence Technique, INRAB, PADSE, p. 48.

Tona Tona A. 2007. Amélioration de la filière canard dans les milieux urbains et périurbains de la ville de Kinshasa République Démocratique du Congo. Mémoire DES, Faculté Universitaire des Sciences Agronomiques, Université de Liège, Genbloux , p. 75.

Vijayakumar P. 1991. Cashew apple utilization: The Nobel method to enhance profit. The Cashew, 5(4): 17-21. 